of time may be ; but vapid hours and aimless days may break up the hardly won discipline of a careful education, if it do not abandon the thoughts to wantonness. The vacuous looks and aimless wanderings of the patients hanging about the precincts of some sanatoriums have impressed me painfully. Vigorous games are rarely suitable; but surely there are occupations, such as gardening, the fine arts, literature, natural history, and quiet handicrafts, which to those free from fever would be beneficial. Perhaps even the visits of teachers in the arts and sciences would not prove so wildly exciting as to throw the population into a fever.

I will sum up the chief questions which I have propounded.

1. Can mixed infections be recognised from fever curves?

2. Can we distinguish between economical (wirtschaftliche) healing and complete (wissenschaftliche) healing? if so, what is the mean term of residence for the economical healing of early cases?

3. How long in certain active cases, say from 6 to 8 per cent, is a febrile patient to be kept to bed in the reasonable hope of recovery? For instance, in a public sanatorium are we justified in retaining patients who have been confined to bed for six months, five months, or even for four months?

4. What estimates of improvement and what rules of prognosis can be based upon physical signs alone?

5. Is multiple tuberculosis, for instance, in lung and testicle too hopeless a condition for a public sanatorium? How far is it comparable with an equal extent of mischief in one organ?

6. Of what use, if any, is massage ?

7. Of what use, if any, is hydrotherapy ?

8. Are special pulmonary exercises appropriate at certain stages of progress? and if so, when, and under what conditions?

9. Must we repair the body at the expense of the life of the mind? Can we not give even some educational value to the sanatorium besides the medical drill of it?

\section{A FURTHER CONTRIBUTION ON ACUTE DILATATION OF THE STOMACH,}

WITH AN ACCOUNT OF TWO ADDITIONAL CASES.

By CHARLES R. BOX, M.D. LoNd., F.R.C.S. ExG., ASSISTANT PHXSICIAN TO ST. THOMAS'S HOSPITAL AND TO THE LONDON FEVER HOSPITAL; AND

CUTHBERT S. WALLACE, F.R.C.S. ENG.

ASSISTANT SURGEON TO ST. THOMAS'S HOSPITAL AND TO THE EAST LONDON HOSPITAL FOR CHIIDREN.

WE have been led by the perusal of a paper on this subject by Dr. Campbell Thomson ${ }^{\prime}$ and the discussions and letters which have recently appeared on the subject to make this addition to a paper published by us in the Transactions of the Clinical Society of London in 1898. In this paper we tabulated and briefly abstracted 16 cases of this rare and fatal condition and reported two new cases, one by the courtesy of Mr. Walter Edmunds and one which had come under our own observation. Since the paper was published other cases with quite typical symptoms and running fatal courses have been recorded by W. H. Brown, ${ }^{2}$ Kirsch, ${ }^{3}$ and T. B. Appel. ${ }^{4}$ Abstracts of these cases may be found in the Medical Revien of the same year, with a reference to a case published by Fenger. ${ }^{5}$ These and additional cases are also given in Mayo Robson and Moynihan's work on Diseases of the Stomach. An attempt was also made to collect cases of the same disease which did not prove fatal. This was difficult, but we found and abstracted the accounts of five cases which appeared to be instances of the disease in question. Care was taken to exclude all cases in which mechanical obstruction at the pylorus was present. Although it appeared to us at the time that the same train of symptoms might follow in cases of this kind, yet we felt that in discussing the cause, symptoms, and diagnosis of the disease it was better to deal with uncomplicated cases only. The two additional cases which we wish to record are as follows.

I'L'HE LANCEX, UCt. ZOUH, 1YUL, p. L115.

3 Deutsche Medicinische Wochenschrift, August 17th, 1899

4 Philadelphia Medical Journal, August 12th, 1899.

5 Clinical Review, 1898.
CASE 1. Lacerated wound of the knee-joint; oellulitis of the leg and thigh; ultimate amputation of the limb; acute dilatation of the stomach and of part of the duodenum.-The patient, a male, aged 29 years, came under observation in 1900 with a lacerated wound of the left knee, opening the joint. A large amount of grit was ground into the wound. The injury was the result of a railway crush, the patient attempting to enter a train in motion and his leg being dragged between the footboard and the platform. The joint wound gave considerable trouble, pockets of pus forming both above and below the joint. Later an arthrectomy was performed and ultimately, on account of severe and repeated hremorrhage, it became necessary to amputate the thigh. This was about a month after the accident. The patient at the time was extremely collapsed and hardly responded to intravenous infusion of saline fluid, or to brandy or strychnine injections. The bowels were not confined but there was no marked diarrhœea. Unfortunately, no note was made as to the presence or absence of vomiting.

The post-mortem examination was made $17 \frac{1}{2}$ hours after death. The only marked visceral abnormality was the condition of the stomach and duodenum. The stomach was dilated and was very prominent. Its upper border was not displaced. Its lower border extended well below the umbilicus. The dilatation was not confined to the stomach, for the first part of the duodenum was dilated to twice its normal size. The dilatation extended to the place where the duodenum came in front of the lumbar spine. The stomach contained a little fluid and some odourless gas.

CASE 2. Pleuro-pneumonia with severe toxic symptoms; acute dilatation of the stomach and duodenum.-The patient, a man, aged 24 years, had previously had no serious illness and came of a healthy family. On May 27th, 1901, after playing cricket, he shivered and shortly afterwards noticed a pain in his left side which caused a "catch" on breathing. On the third day of the illness he was admitted to St. Thomas's Hospital, evidently very ill and with a temperature of $104^{\circ} \mathrm{F}$. Over the lower lobe of the left lung percussion resonance and vocal fremitus were diminished, the breath sounds were very feeble, and a few crepitant sounds were audible. The abdomen was not distended, the abdominal respiratory movements were present, and the abdominal viscera were apparently normal. On the fourth day of the illness the temperature suddenly fell to $96^{\circ}$, but it rose again the same evening to $102^{\circ}$, and steadily mounted from that time until death when it reached $104^{\circ}$. In addition to the signs mentioned at the base of the left lung, a patch of faint tubular breathing developed in the left scapular region and rhonchi and moist sounds were present over the other parts of the lungs. Profuse and uncontrollable diarrhoea now set in, the bowels acting 15 times on the eighth, five times on the ninth, and 11 times on the tenth day of the disease. The patient was sleepless, complained of severe epigastric pain, and commenced to vomit. He was so collapsed that saline fluid was injected into the subcutaneous tissues, brandy being administered freely and strychnine being injected. Death occurred 11 days from the commencement of the disease. Up to the day before death the urine contained no albumin. No urine measurement was made.

At the post-mortem examination the body was found to be emaciated, the trachea and bronchi contained a large quantity of tenacious mucus, the bronchial glands were swollen, and the left lung was completely pneumonic, the lower lobe being grey and œdematous whilst the upper was still red. The visceral pleura was covered with a thick fibrinous pellicle. The lower lobe of the right lung showed patchy grey hepatisation; the other lobes were congested and odematous. There was a fibrinous pellicle on the pleural surface of the lower lobe of this side. The heart was not dilated; the cavities of the right side contained tough fibrinous clot, which extended from the auricle to the commencement of the pulmonary artery. There was no endocarditis and no excess of pericardial fluid. The parietal pericardium, however, was thickened and adherent to the superjacent pleura of the left side. The adhesions were recent and "buttery." Inflammation had not spread to the interior of the pericardial sac. The stomach was greatly distended. It lay with its long axis directed downwards and to the right. 'The lowest part of the viscus was within a few inches of the pylorus and reached the level of a line joining the anterior superior iliac spines. The lesser curvature, after emerging from beneath the liver, ran almost vertically downwards to a point on the right of the mid-line on 
a level with the umbilicus, then becoming sharply recurved and ascending to the pylorus. The distension was not limited to the stomach but involved also the whole of the cluodenum, stopping short near the jejunum. The interior of the stomach showed no gross disease ; its contents were partly fluid and partly gaseous. The intestines below the duodenum were partially collapsed. The liver appeared to be fatty, the spleen was firm and slightly enlarged, and the kidneys were somewhat swollen, probably as the result of tubal changes. The portal and biliary systems were natural. There was no peritonitis. (For the clinical notes of this case we are indebted to the courtesy of Dr. Hector Mackenzie.)

In our previous paper we particularly drew attention to the fact that the dilatation was not neccssarily limited to the stomach but in four of the cases involved the duodenum as well, and in one of these four the first part of the jejunum was said to be also dilated. In both the cases we now record the duodentm was dilated, in one a part ancl in the other practically the whole of the duodenum being involved. In one of the cases lately recorded by Dr. Campbell Thomson a similar condition of affairs was noted. In the face of this evidence it seems impossible to maintain any longer, as some apparently still do, that the dilatation is due to pyloric spasm. The presence of bile in the stomach contents is additional evidence against spasm of the outlet of the stomach.

One point was very noticeable at the operation on our previous and first case: it was that the stomach was so tensely distended that it actually emitted a musical sound when an attempt was made to pick up its wall. Now it is impossible to believe that a paralytically distended viscus could be placed in a condition of tension so yreat as this without some mechanical obstructive factor. The question is, How is the obstruction produced? So far as we can gather from literature the advocates of mechanical obstruction of the duodenum incline to one of two views-either the duodenum is kinked by depression of the distended stomach or it is compressed by the root of the mesentery and superior mesenteric vessels which cross it. The advocates of the latter view postulate a primary collapse of the intestine and prolapse of the empty coils, such as to cause dragging on the root of the mesentery and superior mesenteric vessels.

We have found by actual experiment on the cadaver that the stomach can be enormously distended by water pressure, with the jejunum cut right across and lying patent in the abdomen. Moreover, the stomach remains thus distended. The same result can be attained after the superior mesen. teric vessels and the peritoneal folds in their neighbourhood have all been divided. If, however, by introduction of the finger well behind the distended stomach a little to the left of the mid-line of the spinal column the fundus and posterior wall of the stomach be gently raised the excess of fluid will at once flow freely away from the stomach through the cut jejunum. If the part of the duodenum which lies on the right side of the spine, behind the peritoneum, be first incised, the tense distension of the stomach cannot be produced. We therefore feel justified in assuming that the tense distension is due to actual pressure of the stomach on the part of the duodenum which crosses the third and ascends by the side of the second lumbar vertebra to end in the jejunum.

We would suggest, therefore, that in producing the train of symptoms met with in acute dilatation of the stomach two factors come into play. There is first a paralytic condition of the viscus which leads to distension, and then, at a certain stage the distended stomach actually produces obstruction by pressing on the duodenum on the front and to the left of the spinal column.

The persistence of the vomiting is often held to controvert the idea that the primary lesion is paralytic. It is well to bear in mind the present teaching of physiology on this question. Vomiting may be induced by the injection of tartar emetic into the blood, even after the stomach has been replaced by a bladder, provided that the cardiac orifice has also been removed so that the blaclder is actually tied into the cesophagus. If the cardiac orifice be left intact vomiting cannot be induced. Two factors are therefore essential for the production of vomiting: (1) a dilatation of the cardiac orifice and (2) compression of the stomach by the abdominal muscles and diaphragm or in some cases by the diaphragm only. The presence of the power to vomit is not therefore evidence that the stomach is not paralysed.

Acutely distended stomachs usually contain a quantity of gas as well as of bilious fluid. It is quite possible that a large quantity of this usually, but not always, odourless gas is derived from air which is consciously or unconsciously swallowed, and helps to increase the gastric distension. At the same time saliva is also swallowed, the patient's thirst leads him to drink freely, and possibly gastric hypersecretion also comes into play. When vomiting ceases in cases of acute dilatation of the stomach apparently the abdominal distension becomes extreme.

The early diagnosis of the affection is a point of no little importance. Treatment is apparently futile when the distension becomes extreme. It remains to be seen what can be effected by judicious management in the earlier stages of the affection. A consideration of the recorded cases shows that the recognition of the conclition is by no means easy. It has been mistaken for (1) intestinal obstruction, (2) perforative peritonitis, (3) perforation of bowel with encysted abscess, (4) pancreatic cyst, (5) uræmia, and (6) postanæesthetic vomiting. 1. Of obstruction it doubtless is form, but of obstruction high up, with obtrusive abdominal signs in the form of gastric dilatation. It must be noted, however, that diarrhcea is present and severe in some cases. 2,3 , and 4 . The intense abdominal pain which is usually present, the vomiting, the increasing abdominal distension, and the very marked collapse closeiy mimic general peritonitis. The position of the percussion dulness in the lower part of the abdomen and right iliac region may on a superficial examination appear to confirm this suspicion. The enormously distended stomach has even been mistaken for an encysted abscess. 5. The incessant vomiting, the partial suppression of urine, the occasional albuminuria, the condition of somnolent delirium which may mark the approaching termination, and the "collapse" temperature which is sometimes but not always present, may well give rise to suspicions of uræmia. 6. In those cases which occur after operations there is a great danger of attributing the vomiting to the anesthetic and the collapse to the operation. Careful examination of the abdomen in these cases is the only safeguard.

In all cases the readiest way to a diagnosis lies in a knowledge of the conditions under which acute dilatation of the stomach is likely to occur and in a careful physical examination of the abdomen with an attempt to elicit the succussionsplash.

We pointed out in our other paper that the gastric dilata tion may not at the onset of symptoms assume the extreme degree seen in the later stages of the disease, and that even a day or two may elapse before dilatation is recognised.

It must be borne in mind that the course of the temperature is variable and is largely influenced by the accom panying disease ; a "collapse" temperature is not necessarily present.

The general lines of treatment by strychnine injection, stomach lavage, rectal feeding, and intravenous or rectal saline infusion, have been insisted upon again lately. With regard to an attempt to remedy the obstruction by the postural method we can only say that, we have not been able to satisfy ourselves that it is successful in the cadaver, even with the abdomen freely opened, so that the stomach might fall forwards easily. It would appear rational, however, to adopt the right-sided or prone position after syphonage. It might be advisable also to try the effect of atropine injection, this method being believed by some to be of utility in the cardio-gastric crises of diphtheria. In these, however, obvious dilatation of the stomach is a very uncommon occurrence. Should operation be determined upon the ideal proceclure would certainly appear to be gastro-jejunostomy, as proposed by Mayo Robson in his remarks on the subject. ${ }^{6}$

\section{BRIEF NOTES OF A FEW EXCEPTIONAL CASES OF CATARACT EXTRACTION.}

\section{BY CHARLES BELL TAYLOR, M.D. EDIN.}

SURGEON TO THE NOTTINGHAM AND MIDLAND EYE INFIRMARY.

CASE 1. - This patient, from Middlesbro', was born with opacity of the lens in each eye, not the ordinary form of congenital cataract with an opaque centre and clear margin, but with an opacity affecting the lens throughout, slight at first and not sufficient to prevent his learning to read and to write, but gradually increasing: 